\title{
Light the way home
}

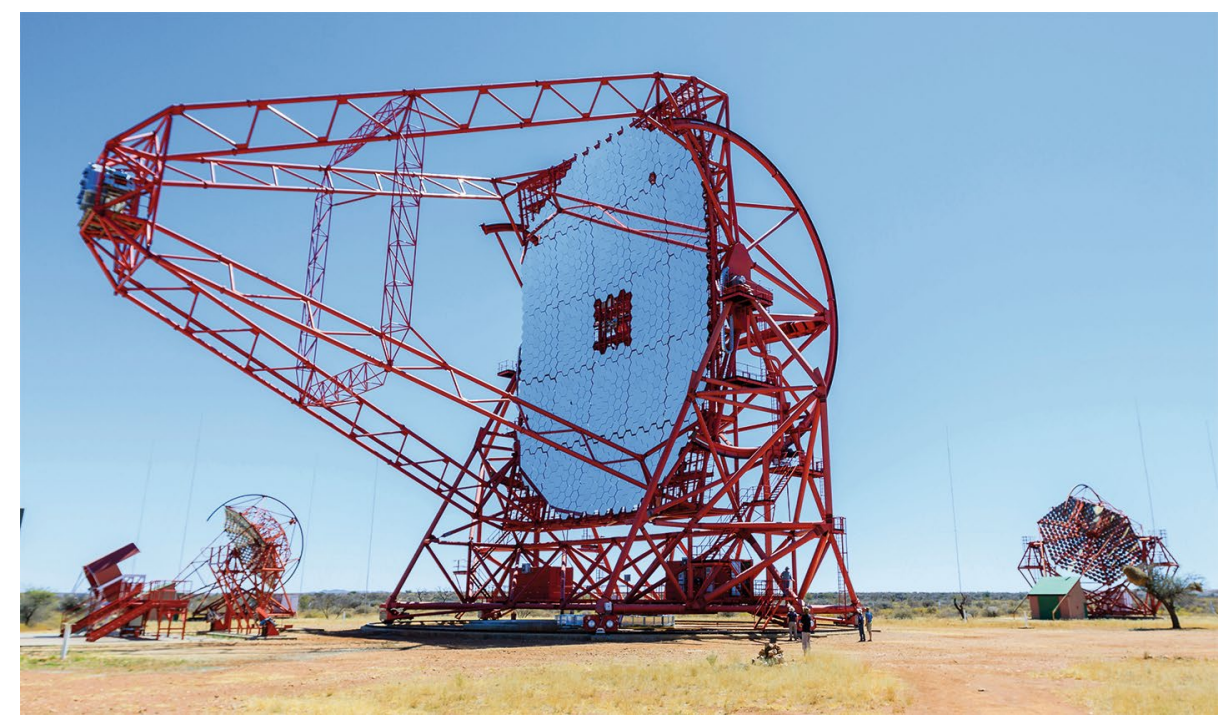

Credit: imageBROKER / Alamy Stock Photo

Natural cosmic particle accelerators can boost charged particles to very high energies using electromagnetic fields, but the mechanistic details remain elusive. And as magnetic fields deflect cosmic rays as they travel, locating their sources can be tricky. Detecting the gamma-rays produced when such cosmic rays interact with matter is more helpful, as photons travel undeflected from their source. To systematically study the production and propagation of energetic particles, the High Energy Stereoscopic System (H.E.S.S.) - five Cherenkov telescopes in Namibia - collected observations of high-energy gamma-rays (up to $100 \mathrm{TeV}$ ) for more than a decade.

The collaboration published their extensive Galactic plane survey and analysis in 14 papers within one issue of Astronomy \& Astrophysics. The telescopes actually measure the Cherenkov radiation emitted by relativistic particles generated when the incoming gamma-rays strike atmospheric molecules. In addition to identifying 16 previously unknown sources, the sky maps and catalogue of 78 sources are a valuable resource for characterizing the diverse type and morphology of these extreme environments, which include pulsars and pulsar wind nebulae, supernova remnants, black holes, micro-quasars and active galactic nuclei. The completed H.E.S.S. survey will shape the strategy for the next gamma-ray observatory, the Cherenkov Telescope Array, with more than 100 telescopes across two sites on different hemispheres.

\section{May Chiao}

Published online: 18 April 2018 https://doi.org/10.1038/s41550-018-0474-4 